\title{
Effect of Ankle Kinesio Taping on Dynamic Balance in Stroke Patients- A Pilot Study
}

\author{
Megha Mohandas ${ }^{1}$, Rutika Patil ${ }^{2}$, Ajay Kumar ${ }^{3}$ \\ ${ }^{1}$ B.P.Th DPO's NETT College of Physiotherapy, Thane, India. \\ ${ }^{2}$ M.P.Th Assistant Professor, DPO'S NETT College of Physiotherapy, Thane. \\ ${ }^{3}$ Principal, DPO's NETT College of Physiotherapy, Thane. \\ Corresponding Author: Megha Mohandas
}

DOI: https://doi.org/10.52403/ijhsr.20220105

\begin{abstract}
Background: Stroke causes partial brain loss, which leads to a functional abnormality of the brain, which produces a variety of symptoms the most prevalent of which is balance affection. Kinesio tape is a thin, light and elastic material which does not immobilize the joint and allows for free movement irrespective of the tape. It serves to facilitate or inhibit the muscle as needed and provides joint stability which will help improve balance function. It is also used to reduce pain, which is a typical complaint in stroke patients.

Objective: To assess for immediate and post effects of kinesio tape in stroke patients using Berg Balance Scale and 10 Meter walk test.

Method: A total of 15 participants were chosen based on inclusion and exclusion criteria. Before application of kinesio tape, the Berg balance scale and a 10-meter walk test were performed and recorded. Following the application, the subjects were reassessed, and the outcomes of the 5-day follow-up were recorded. These scores were statistically analyzed.

Result: The statistical analysis showed that there is a significant increase in the dynamic balance function post kinesio tape application. An average increase of 5 scores in BBS was noted and average improvement of 5.70 seconds in 10MWT.

Conclusion: This study concludes that there is a significant improvement on dynamic balance after application of kinesio tape in stroke patients.
\end{abstract}

Keywords: Stroke, kinesio tape, dynamic balance

\section{INTRODUCTION}

Stroke is a central nervous system disease that causes partial brain loss and functional impairment as a result of the blood vessels' inability to provide blood to the brain. It is said to be rapidly developing clinical signs of cerebral functions with symptoms lasting 24 hours to longer leading to death. The two main type of stroke are A) Ischemic stroke which is caused due to blocked artery which leads lack of blood supply. It is the most frequent form, involving around $80 \%$ of stroke patients, and occurs when a clot blocks or inhibits blood flow, depriving the brain of oxygen and vital nutrients. B) Hemorrhagic stroke which is caused when a blood vessel bursts, resulting in bleeding. (1)(2)

Few people may have transient ischemic attack (TIA), which is a temporary disruption of blood flow to the brain with symptoms that last no more than 24 to 48 hours. Most stroke patients typically experience motor dysfunction and show an abnormality in standing because of asymmetric alignment and posture, abnormal balance ability of the paralyzed muscles due to loss of muscle control which affects the patient's daily activities and quality of life (3)(4) $^{(4)}$ 
Balance is one of the commonly observed problems in stroke patients, which seriously affects the patient's daily activity. According to the severity of stroke, the manifestations of balance impairment can be classified into static and dynamic balance. ${ }^{(5)}$ Due to which walking ability is also affected and has being reported as a limitation of the lower extremities in patients after stroke, including the inability to dorsifex the ankle, ankle instability, slower gait velocity, and increased risk of injury due to frequent fall.

Kinesio taping (KT), which was invented in Japan by Dr. Kenzo Kase and has recently been quite popular in pain therapy, has become increasingly popular. It is a thin, light and elastic material which does not immobilize the joint and allows for free movement irrespective of the tape. Although the exact mechanism of KT is not understood-proprioceptive feedback mechanisms, inhibitory and excitatory nociceptive stimuli, mechanical restraint were explained as underlying mechanism. ${ }^{\text {(3) }}$

It has been found to be effective not only in reducing pain, but also in increasing range of motion (ROM), decreasing muscular spasm, improving local blood supply and lymph circulations, reducing edema, strengthening weakened muscles, controlling joint instability, and maintaining postural alignment.

The benefits of Kinesio Taping (KT) can be combined with other treatments and ensure safety because it is a non-invasive, side-effect-free procedure. (3) Kinesio taping, also called elastic therapeutic tape or elastic sports taping, differs from other types of strapping tapes due to its unique elastic properties. Kinesio taping has gained favour in the treatment of a variety of additional musculoskeletal conditions in recent years. A study showed that the kinesio taping method may help by facilitating or inhibiting muscle function and support joint structure for the upper or lower extremity in hemiplegia in conjunction with other therapeutic interventions. ${ }^{(5)(6)}$
KT is also well-known to be effective for increasing functional movements by improving muscle endurance. It also helps to maintain the coordination of agonist, synergist, and antagonistic muscles by controlling muscle tones, inducing body balance and muscle control recuperation. Thus, balance and muscle control recuperation through KT can increase dynamic balance. ${ }^{(3)(5)}$

The use of kinesio taping for rehabilitation of stroke patients might provide a theoretical basis and practical guidance. The tibialis anterior and the gastrocnemius muscle, play important role in the balance control of stroke patients, when tape is applied to the ankle joint and other two muscles, there might be a significant improvement observed by assessing stroke patients' dynamic balance and gait ability. ${ }^{(3)}$ As a result, an effort is undertaken to ascertain the effect of ankle kinesio tape on dynamic balance in stroke patients.

\section{MATERIAL AND METHODS}

The study was and interventional study where 15 stroke patients were selected using convenient sampling. Inclusion criteria: Subjects must be willing to participate, and they must be post-stroke patients with muscle tone ranging from 1 to 3 on the Modified Ashworth Scale (MAS) and voluntary control of the lower extremities at stage four or higher on the Brunnstrom stages of recovery, with or without knee or ankle pain. Exclusion criteria: Fracture, amputation, skin infection, Deep Vein Thrombosis (DVT).

\section{PROCEDURE:}

Institutional ethical committee clearance was taken. A written consent was taken from all the willing participants in the language best understood by them. Selection of the subjects was done as per the inclusion criteria and exclusion criteria. The protocol and goal of the study were explained to the subjects prior to the start of the study. Subject's dynamic balance and gait was 
assessed using Berg Balance Scale and 10 Meter Walk Test. After assessing the subject application of the kinesio tape was done. Immediate post balance was checked using the same scales and 5-day follow up was done where the dynamic balance was again re-assessed using the same scales. Data was collected and statistically analysed using SPSS 28.0.0.0

\section{METHOD OF APPLICATION OF KINESIOTAPING:}

A] K-Taping for tibialis anterior:

Position: Patient was in supine position with knee flexed.

Method: I-strip K-Tape was applied from origin to insertion- from lateral surface of tibia to medial cuneiform bone in order to facilitate the muscle. Tape was anchored with no tension to distal ends and 15-25\% tension was applied mid-way through the tape.

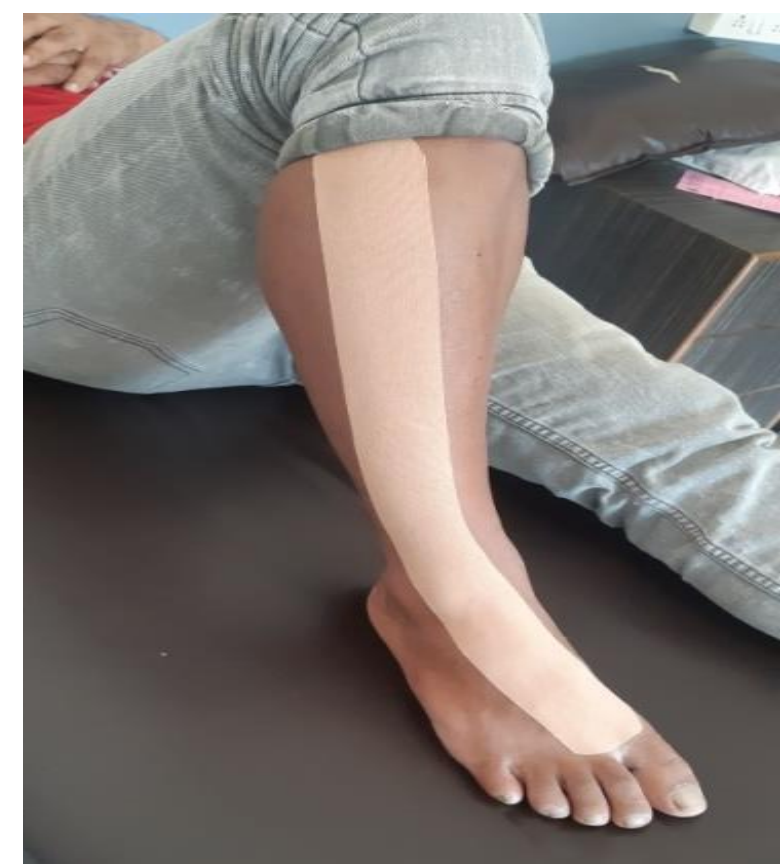

Fig. 1: Kinesio taping for tibialis anterior muscle

\section{B] K-Taping for gastrocnemius:}

Position: Patient was in supine position with knee flexed.

Method: Y-strip K-Tape was used from the origin to the insertion- for the lateral headposterolateral aspect of the lateral condyle of the femur and for medial head- posterior surface of medial femoral condyle to posterior surface of the calcaneus in order to facilitate the muscle. Paper off tension or $15-25 \%$ tension was applied mid-way through the tape and distal ends with no tension.

\section{C] K-Taping for ankle stability:}

Position: Patient in supine position with lower extremity extended and ankle at the edge of the plinth.

Method: Figure of eight taping method was used to apply for the ankle stability. Only paper off tension was applied in this method.

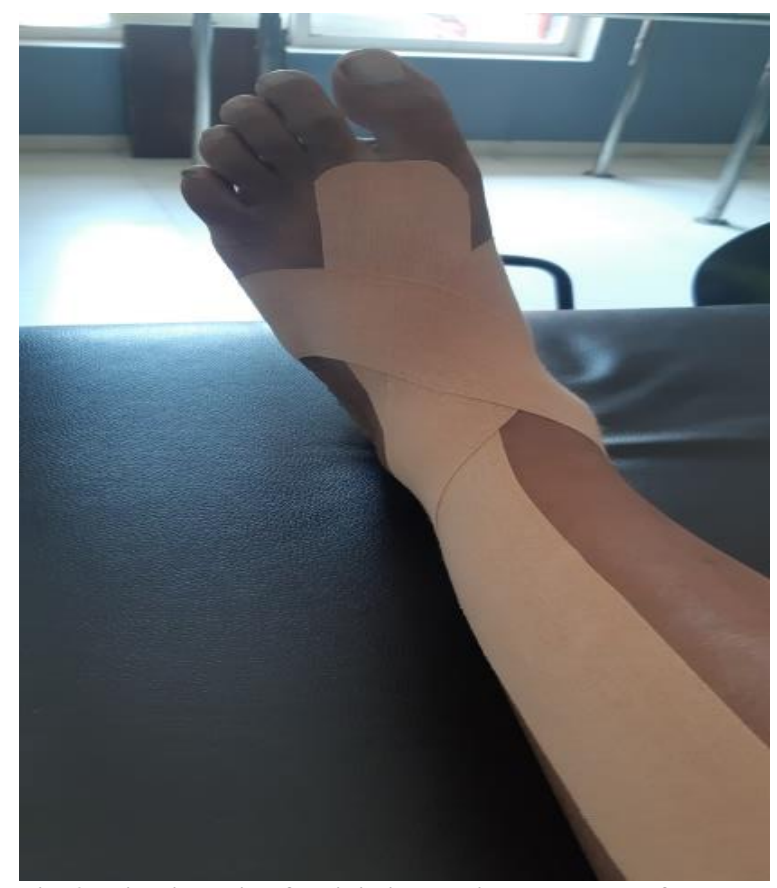

Fig. 2: Kinesio taping for tibialis anterior muscle and for ankle stability

\section{STATISTICAL ANALYSIS:}

All data was analysed using SPSS version 28.0.0.0. Normality test ShapiroWilk test was done to assess whether the data was normally distributed. Results of the test revealed that the data was not regularly distributed. Hence, a Non parametric test Wilcoxon signed rank test was used to assess the pre-test and post-test of 10 MWT and BBS. Their significance levels are $\mathrm{p}$ value 0.001 .

\section{RESULTS}

From 15 participants in the study, 7 were males and 8 were females. Age range 
was 50-72 years, with an average of $61.2( \pm 7.0)$ years. BBS and 10 Meter Walk test was done for all the 15 participants and when statistically analysed using Wilcoxon test, the BBS scale comprising total of 56 score and showed maximum value of 54 post application of kinesio tape for dynamic balance of stroke patients an average increase of 5 scores in BBS was noted and average improvement of 5.70 seconds in 10MWT. The Wilcoxon signed rank test analysed the Berg Balance Scale and 10 Meter Walk Test to show statistically significant improvement, with both having p- values $<0.001$.

\section{Tests Of Normality}

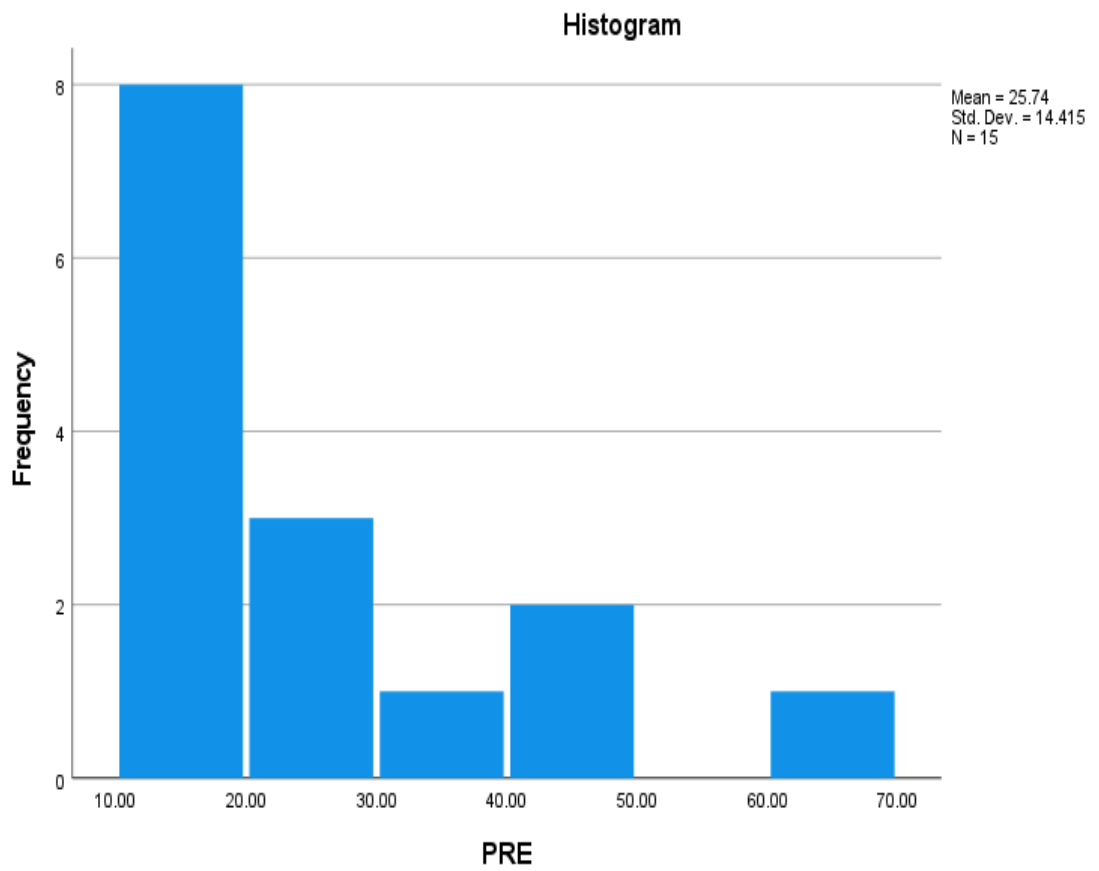

Shapiro-wilk test was done to assess whether the data is normally distributed. The test showed that the data was not normally distributed and shows $(\mathrm{p}<.05)$ and visual inspection of histograms showed that the value are not normally distributed, with a skewness of $1.630 \quad(\mathrm{SE}=0.580)$ and kurtosis of 2.498 ( $\mathrm{SE}=1.121)$.

\section{For Berg Balance Scale:}

Table (1)

\begin{tabular}{|l|l|l|l|l|}
\hline & Mean & Std. Deviation & Z value & P value \\
\hline PRE & 37.26 & 6.51 & -3.419 & $<.001$ \\
\hline POST & 42.33 & 5.65 & -3.420 & $<.001$ \\
\hline DAY 5 & 42.20 & 5.78 & -3.420 & $<.001$ \\
\hline
\end{tabular}

Table 1: Demonstrates pre, post and 5 day follow up of the Berg Balance Scale. p- value is <.0.001 and is statistically significant.

\section{For 10 Meter Walk Test:}

Table (2)

\begin{tabular}{|l|l|l|l|l|}
\hline & Mean & Std. Deviation & Z value & P value \\
\hline PRE & 25.7447 & 14.41 & -3.408 & $<.001$ \\
\hline POST & 20.5907 & 10.27 & -3.408 & $<.001$ \\
\hline DAY 5 & 20.1053 & 10.16 & -3.408 & $<.001$ \\
\hline
\end{tabular}

Table 2: Demonstrates pre, post and 5 day follow up of the 10Meter Walk Test. p- value is $<0.001$ and is statistically significant. 


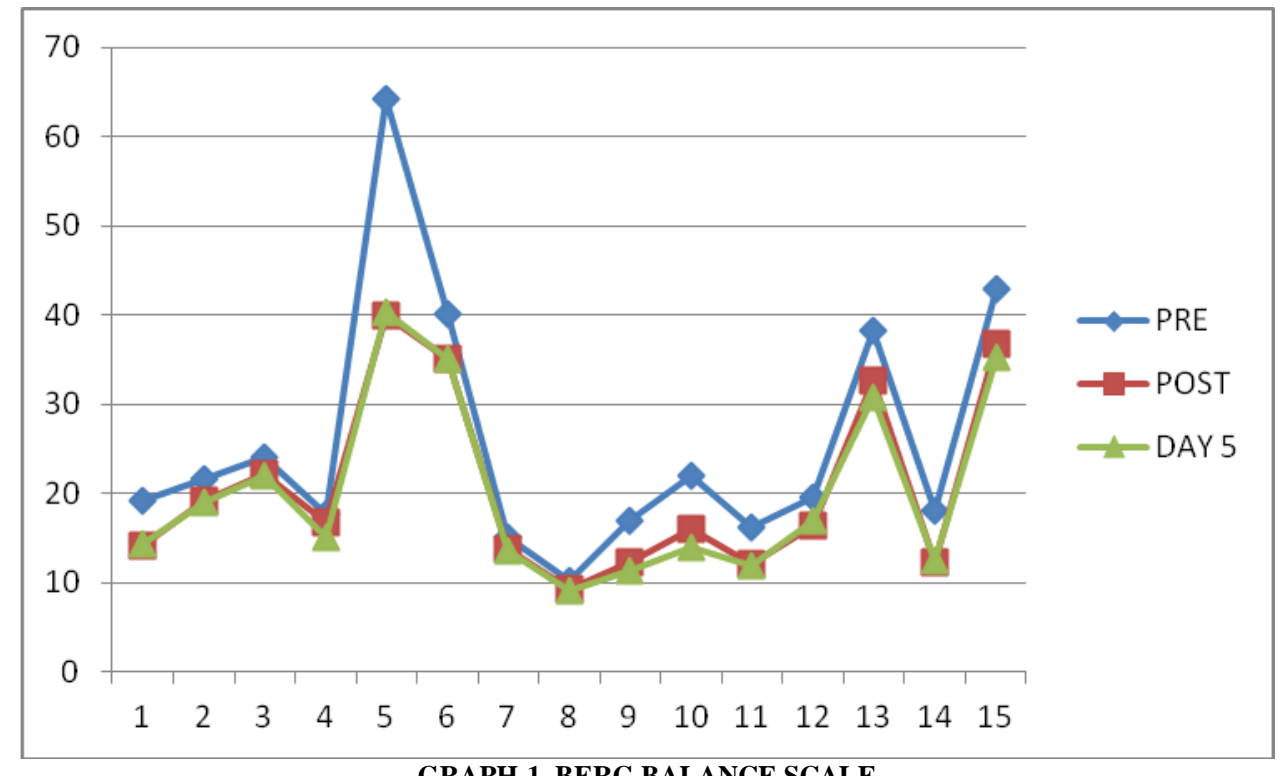

\section{INTERPRETATION:}

In Graph 1- On X-Axis shows population- number of participants is noted and on Y-Axis is the score of the Berg
Balance Scale (BBS). Three graphs are observed which depicts the scoring of individuals in pre, post and $5^{\text {th }}$ day of application of kinesio tape.

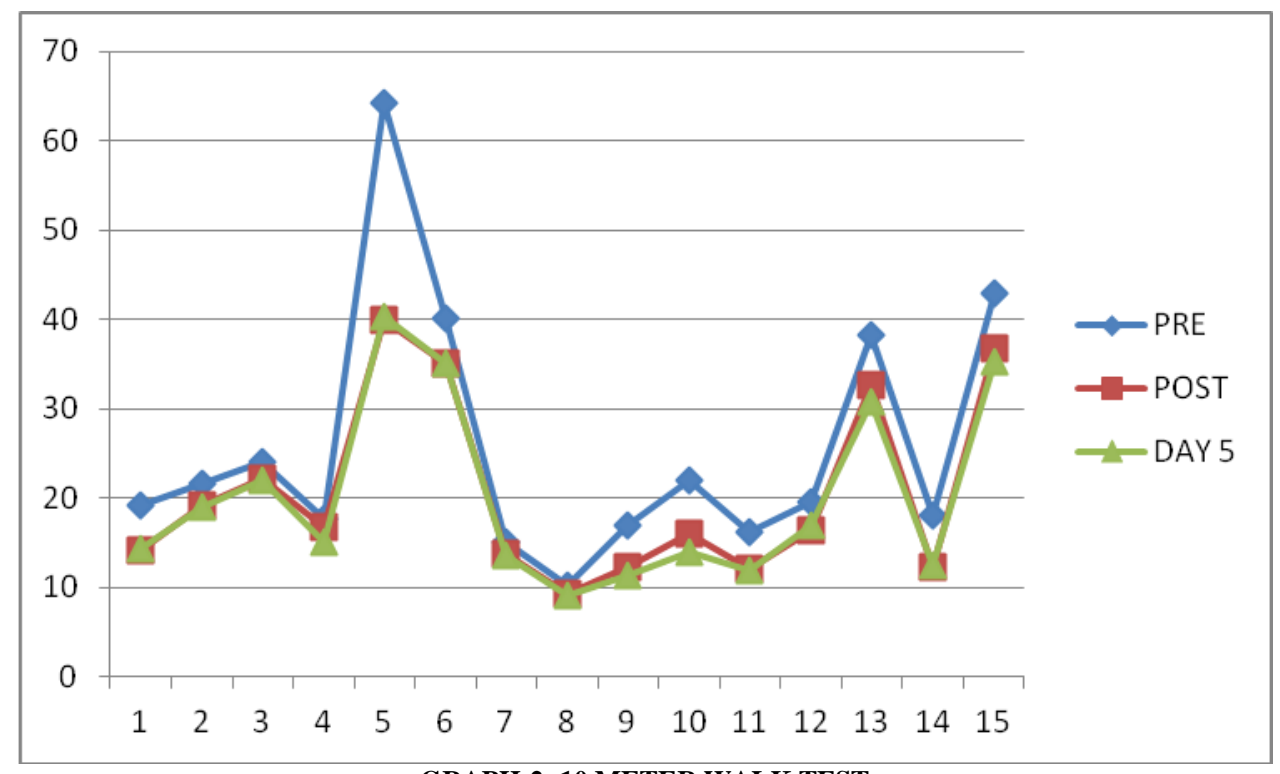

GRAPH-2: 10 METER WALK TEST

\section{INTERPRETATION:}

In Graph 2- On X-Axis shows population- number of participants is noted and on Y-Axis is the time recorded in 10 Meter Walk Test. Three graphs are observed which depicts the scoring of individuals in pre, post and $5^{\text {th }}$ day of application of kinesio tape.

\section{DISCUSSION}

Stroke results in abnormal muscle tone, synergy patterns, poor voluntary control. Thus, the function of the affected lower extremity in hemiplegics is diminished. Hemiplegics when asked to perform activities of lower extremity, the movements are not smooth and coordinated. Impaired hip, knee, and ankle joint stability and mobility, 
spasticity/weakness of involved muscles of lower extremity, synergy patterns and pain around joints are likely reasons for this. Thus, bone alignment, joint stability and mobility, kinetic forces produced by muscles, muscle tone, and the lengthtension relationship are all required for the lower extremity's movements and functions to run well. ${ }^{(1)}$

In this study, KT was given to the paralysed lower extremities of stroke patients to see how it affected their gait and balance ability. Brain damage causes hemi paralysis depending on the region in which the damage is located, and 70 to $75 \%$ of patients struggle to recover fully and experience problems such as motor, gait, and sensory impairments, as well as difficulties with activities of daily living. Such conditions limit movement and daily activities, and a lack of mobility in the lower extremities, in particular, has a negative impact on gait and balance. ${ }^{(3)}$

An increase in muscle tone was detected in ankle dorsiflexors, as well as weakening in plantarflexors, resulting in imbalance and instability in stroke patients. In this study, application of kinesio tape to tibialis anterior, gastrocnemius by facilitating both the muscles from origin to insertion helped to increase the balance function. This causes a concentric pull on the fascia, which activates the muscles and stabilises the ankle. There was a significant improvement seen in baseline and post 5 day noted on Berg Balance Scale and 10 Meter walk test. Data was statistically significant with $\mathrm{p}$-value of $<0.001$.

This present study showed improvement in balance by an average increase of 5 scores in BBS was observed and average improvement of 5.70 seconds in 10MWT. The mechanism responsible for the improvement observed is due to the motor neuron pool that involves the cutaneous receptors. The application of kinesio tape did not result in a specific reduction in spasticity, but it did offer ankle stability at neutral and also facilitation of the ankle plantarflexor and dorsiflexor was observed which thus helped to reduce the time taken to cover the 10 meters and also to perform the components of berg balance better.

No significant change was seen in co-ordination and sensation but time taken to perform the activity like sit to stand, 360 degree turn and retrieving object from the floor as described in the berg balance scale was quicker and even easier after application of KT. Some components of Berg balance scale such as placing alternate foot on stool, standing with one foot in front, standing on one foot showed no difference irrespective of the application of KT.

Our study differs from others in this way because it included younger age groups and the method of application to test balance was previously used more widely for the core muscles. ${ }^{(9)}$ Several studies have used different outcome measures were used such as forward reach test, Romberg test, Time Up and Go test and Dynamic gait index.

Page et al. reported that the treatment for symmetry can help stroke victims regain their functional independence. From this, it can be deduced that direct activation of muscles in the paralysed areas of the extremities can enhance balance and walking abilities. ${ }^{(3)}$

Previous studies conducted in other countries like Japan and Korea among not just stroke patients but also patients with foot drop were included. In a study conducted by Woo-Il Kim, to investigate the effects of KT applied to the upper and lower extremities on asymmetric gait, walking speed, and balance ability in stroke patients. Application of KT to the paralyzed parts of a stroke patient had a positive effect not only on improvement walking speed but also of typical gait pattern (asymmetric gait). (3)

However, a study done by Zack $\mathrm{M}$ Slevin et al it was seen that kinesio taping for ankle joint did not show any significance on their outcome measure. They also compared the findings of $\mathrm{k}$-tape to the 
stability provided by a fitted shoe which seemed to be more beneficial. Whereas, our study showed significance of ( $p$-value $<0.001)$, this could be because the muscle which were considered for the application of tape was different. KT was applied to tibialis anterior and peroneus longus muscle.

Another study by Yang Rae Kim et al found that ankle kinesio taping did not have much statistical value on its own. The possible reasons for this may be that Yang Rae Kim et al. combined too many tests together - Romberg's eye open test, Romberg's eye close test, Limit of stability, Forward and back test, Timed Up and Go test, $10 \mathrm{~m}$ gait velocity test, which certainly contributed to heterogeneity. ${ }^{(4)}$

In studies where two groups were taken into consideration, the group where in taping was combined with therapeutic exercises showed more improvement as compared to the other group.

Huang YC et al. showed positive effects ok KT on hemiplegic pain. In this study too, pain was reduced after KT application and as stated by dos Santos GL et al., it can be suggested that a possible explanation for the effect of elastic taping may be related to the neural activation (mechanoreceptors) and biomechanics support. ${ }^{(8)}$

By applying KT to lower limb muscles the over activated muscles are attributed to be inhibited and weak being facilitated which in turn helped in lower extremity movements an, balance and mixed synergies and also mainly providing stability to ankle joint for the activities like walking. Thus, KT should be considered as an adjunct to the functional training given for the ankle to be focused in post-stroke hemiplegics.

As the previous studies have been more related to static balance, our study inculcates the effect of kinesio tape in stroke patients.

\section{Limitation}

Small sample size was taken which was due to the study being conducted during the covid-19 pandemic.

\section{CONCLUSION}

This study concludes that there is a significant improvement on dynamic balance after application of kinesio tape in stroke patients.

\section{Abbreviations \\ KT: Kinesio taping \\ 10MWT: 10 Meter walk test \\ BBS: Berg balance scale}

\section{Acknowledgements}

I express my deep sense of gratitude and sincere thanks to our respected sir Dr, Ajay Kumar and my guide Dr. Rutika Patil who immensely helped me with sincere guidance, co-operation, valuable advice and endless inspiration during course of study. I also take this opportunity to thank teaching and non-teaching staff, my friends and colleagues for their support.

\section{Conflict of Interest: None}

\section{Source of Funding: None}

\section{Ethical Approval: Approved}

\section{REFERENCES}

1. O'Sullivan, Susan B.; Schmitz, Thomas J.; and Fulk, George D., "Physical Rehabilitation, 6th edition" (2014).

2. Y. Munjal, S. Sharma, M. A. K. Agarwal, and P. Gupta, Api Textbook of Medicine, Series G: Reference, Information and Interdisciplinary Subjects Series, Jaypee Brothers, Medical Publishers, 2012.

3. Woo-Il Kim, Yong-Kyu Choi, Jung-Ho Lee, Young-Han Park. The Effect of Muscle Facilitation Using Kinesio Taping on Walking and Balance of Stroke Patients. J. Phys. Ther. Sci. (2014) 26: 1831-1834.

4. Yang Rae Kima, Jae Ic Kimb, Yong Youn Kimc, Kwon Young Kangd, Bo Kyoung Kime, Joo Hyun Parke et.al Effects of Ankle Joint Taping on Postural Balance 
Control in Stroke Patients. Int Acad Phys Ther Res.(2012) 3(2): 413-478

5. Wang M, Pei ZW, Xiong BD, Meng XM, Chen XL, Liao WJ. Use of Kinesio taping in lower-extremity rehabilitation of post-stroke patients: A systematic review and metaanalysis. Complement Ther Clin Pract.(2019)

6. Yilan Sheng, Shifeng Kan, Zixing Wen, Wenhua Chen, Qi Qi, Qiang Qu et.al. Effect of Kinesio Taping on the Walking Ability of Patients with Foot Drop after Stroke.(2019)

7. $\mathrm{Hu} \mathrm{Y}$, Zhong $\mathrm{D}, \mathrm{Xiao} \mathrm{Q}$, Chen Q, Li J, Jin R. Kinesio Taping for Balance Function after Stroke: A Systematic Review and Meta-Analysis. Evid Based Complement Alternat Med. (July 2019) 8470235.

8. Huang YC, Leong CP, Wang L, Wang LY, Yang YC, Chuang CY, Hsin YJ. Effect of kinesiology taping on hemiplegic shoulder pain and functional outcomes in subacute stroke patients: a randomized controlled study. European journal of physical and rehabilitation medicine. (Dec 2016) 52(6):774-81.

9. Yang Jin Lee, Ji Young Kim, Seonng Yoel Kim. The effects of trunk kinesiotaping on balance ability and gait function in stroke patients. Journal of physical therapy science.(2016)

10. Sarvestan J, Svoboda Z. Acute Effect of Ankle Kinesio and Athletic Taping on Ankle Range of Motion During Various Agility Tests in Athletes With Chronic Ankle Sprain. J Sport Rehabil. (2019) 26;30(5):689-696

How to cite this article: Mohandas M, Patil R, Kumar A. Effect of ankle kinesio taping on dynamic balance in stroke patients- a pilot study. Int J Health Sci Res. 2022; 12(1): 28-35. DOI: https://doi.org/10.52403/ijhsr.20220105 